\title{
Graphitization of gallium-incorporated carbon nanofibers and cones: in situ and ex situ transmission electron microscopy studies
}

\begin{abstract}
This study demonstrates graphitization directly through the amorphous carbon under the catalysis of a low-melting-point metal, gallium $(\mathrm{Ga})$, by heating in a vacuum heater as well as by Joule heating during in situ transmission electron microscopy (TEM) operation. For the material system of the mixture of Ga nanoparticles (NPs) and amorphous carbon matrix, the graphitization temperature is determined to be about $600{ }^{\circ} \mathrm{C}$ for the first time. With increasing the temperature, evaporation and agglomeration of small Ga NPs start to occur together with the graphitization at around the places where Ga NPs would have been located at the surface region. In situ TEM experiment reveals the accelerated increase in electrical conductivity with structural change from amorphous to graphitization. Thus, the combination of the in situ and ex situ TEM observations is believed to be a lead step to understand deeper the graphitization process and provide information in nanoscale.
\end{abstract}

